San Jose State University

From the SelectedWorks of Juana Vivó Acrivos

1978

\title{
In Situ Intercalation of TaS2 in the Electron Microscope
}

G. J. Tatlock, University of Sussex

Juana Vivó Acrivos, San Jose State University

Available at: https://works.bepress.com/juana_acrivos/30/ 


\title{
In Situ Intercalation of $T_{a} S_{2}$ in the Electron Microscope
}

\author{
G.J Tatlock \\ School of Mathematical \& Physical Sciences, University of Sussex, Falmer, Brighton BN1 92H, England \\ J.V Acrivos \\ Department of Chemistry, San Jose State University, CA 95192, USA
}

\begin{abstract}
$\underline{\text { Abstract }}$
Electron diffraction has been used to study the formation of a variety of complex superlattices in the $1 \mathrm{~T}, 2 \mathrm{H}$ and $4 \mathrm{Hb}$ polytypes of $T_{a} S_{2}$ during intercalation with hydrazine gas $\left(\mathrm{N}_{2} \mathrm{H}_{4}\right)$, in situ in the electron microscope. Results are presented over the temperature range $15 \mathrm{k}-$ $360 \mathrm{k}$ and are interpreted in terms of scattering from periodic lattice distortions linked to change density waves. Comparisons are also made with similar effects in both the as-grown crystals and $T_{a} S_{2}$ samples containing other adducts.
\end{abstract}

\section{Introduction}

Although superlattice effects in high-energy electron diffraction patterns of $T_{a} S_{2}$ were reported by Wilson and Yoffe in (1969), it was not until 1974 that detailed interpretations of this behavior in terms of scattering from periodic lattice distor tions linked to charge density waves were put forward (Wilson et-al 1976, Williams et-al 1974, Scruby et-al 1975, Van Landuyt et-al 1974). Changes in the transport properties of layer structures when adducts are inserted between the layers have also been known for some time but electron microscopy has only been used quite recently to investigate these effects (e.g. Carter \& Williams (1972), Beal \& Liang (1973), Parry et-al (1974), Wilson et-al (1975)).

The advantages of the transmission electron microscope (TEM) for these studies are that the morphology of the crystals may be observed at the same time as diffraction evidence for the existence of superlattices. Weak scattering is also enhanced in the electron diffraction patterns by dynamic mechanisms such as double diffraction. Hence it is much easier to observe subtle changes in the superlattices by electron rather than by x-ray diffraction, for example. Finally, in some cases high resolution lattice imaging may be used to give information about the perfection of the distorted lattices.

In this paper we report new results on the intercalation of the $1 \mathrm{~T}, 2 \mathrm{H}$ and $4 \mathrm{Hb}$ polytypes of $T_{a} S_{2}$ with hydrazine gas $\left(N_{2} H_{4}\right)$. The $T_{a} S_{2} / N_{2} H_{4}$ system is eminently suitable for this type of 
investigation since detailed studies of the as grown $T_{a} S_{2}$ polytypes have already been carried out over a wide range of temperatures (Scruby et al 1974, Tidman et al 1976, Wilson et al 1975 Tatlock 1976). The chemical reactions of $N_{2} H_{4}$ with $T_{a} S_{2}$ have also been studied (e.g. Acrivos et al 1976) moreover since low pressure hydrazine gas is a suitable source of adduct it was possible to perform the whole intercalation experiment, including appropriate heating or cooling, in situ in a cell within the T.E.M. Hence any ambiguities about possible deintercalation prior to examination could be removed.

The experimental procedure is described in the next section while detailed experimental results for the three polytypes are presented in $\$ 3$. Finally the results are interpreted in the light of experiments on other systems in $\$ 4$.

\section{Experimental}

Crystal samples were cleaved with adhesive tape and floated onto electron microscope grids in the usual way. These were then inserted in a cell which was subsequently loaded into an HU11B electron microscope equipped with a heating/cooling stage. The cell was a modified version of the one designed by English \& Venables (1971) for work on condensed gases and is shown schematically in Fig. 1. Electron transparent films at top and bottom enable the cell to be isolated from the column vacuum $\left(\sim 10^{-6} t\right)$ while maintaining a gas pressure of severeal torr inside the cell. Hence a sample can be intercalated in situ in the TEM and subsequently heated or cooled within the temperature range $15 \mathrm{~K}-360 \mathrm{~K}$ during examination. Deintercalation can also be studied by leaking air, water vapor etc. into the cell instead of hydrazine.

\section{Results and Discussion}

\subsection{T Polytype}

The 1T polytype of $T_{a} S_{2}$ exists in the three distinct phases below $400 \mathrm{~K}$ designated $1 T_{1}, 1 T_{2}, 1 T_{3}$ after Williams et al (1974). Resistance measurements such as those of Thompson et al (1971) shown in Fig 2 indicate rapid changes in resistivity on going from one phase to the other. Characteristic diffraction patterns are also obtained for each phase and these were discussed in detail by Scruby et al (1975). At room temperature the $1 T_{2}$ phase gives rise to weak extra reflections in the hk.O pattern shown in Fig 3a at positions linked by wave vectors $\mathrm{Q}$ where $\mathrm{Q} / \mathrm{a}^{*} \sim 0.285$ and $\varnothing$, the angle between $\mathrm{Q}$ and $\mathrm{a}^{*}$, is $12^{\circ}$. Below $190 \mathrm{~K}$, however, $\mathrm{a} \sqrt{13}$ superlattice is present here $\mathrm{Q} / \mathrm{a}^{*} \sim 0.277$ and $\varnothing=13.4^{\circ}$.

Resistivity measurements on $1 \mathrm{~T} T_{a} S_{2}$ intercalated with ethylenediamine by Meyer et al (1974) are also shown in Fig 2 where it can be seen that the $1 T_{2}-1 T_{3}$ transition is smeared out. Hence it 
was thought that room temp intercalation of 1 t $T_{a} S_{2}$ with $N_{2} H_{4}$ or similar adducts, may alter the lattice distortions in the crystal. Fig $3 \mathrm{~b}$ shows the result of such an experiment where a crystal of $1 \mathrm{~T}$ $T_{a} S_{2}$ was exposed to $N_{2} H_{4}$ gas at a pressure of $\sim 1$ Torr for 5 min. A new $\sqrt{7} x \sqrt{7}$ lattice is produced which is analyzed in Fig 4. Although the superlattice could be due to scattering from ordered $\mathrm{N}_{2} \mathrm{H}_{4}$ molecules this seems very unlikely since such scattering would be much weaker than that observed in practice. And from nmr measurements we known the adduct has liquid like motion. Also recent experiments on the intercalate $1 \mathrm{~T} T_{a} S_{2}$ with alkali metals occasionally produced similar patterns (Clarke + Williams 1978). Hence a more likely explanation is a change in the lattice distortion caused by the presence of the intercalate. A much more diffuse $\sqrt{7} x \sqrt{7}$ superlattice has also been reported in $4 \mathrm{Hb} T_{a} S_{2}$ intercalated with ethylenediamine (Wilson et al 1976) and this will be returned to in $\$ 3.3$.

Heating and cooling experiments were also attempted in an effort to obtain further information about the superlattice and the results are sown in Fig 5 . On cooling to $80 \mathrm{~K}$ the $\sqrt{7} x \sqrt{7}$ lattice becomes very diffuse and a commensurate $3 \times 3$ lattice is formed, although the $\sqrt{7} x \sqrt{7}$ returned on warming. The diffraction pattern in Fig $5 \mathrm{~b}$ also shows traces of a $\sqrt{13} x \sqrt{13}$ lattice. Heating the specimen to $360 \mathrm{~K}$ gave the pattern shown in Fig $5 \mathrm{c}$ the $\sqrt{7} x \sqrt{7}$ lattice has given way to an incommensurate distortion with $\mathrm{Q} / \mathrm{a}^{*}=0.288$. On returning to room temperature the $\sqrt{7} x \sqrt{7}$ was replaced by a $\sqrt{13} x \sqrt{13}$ lattice similar to the one observed at room temperature in the as grown $4 \mathrm{Hb}$ polytype; and this seemed to be the stable end product of all the reactions in $1 \mathrm{~T}$ intercalated with hydrazine.

\subsection{H Polytype}

As grown $2 \mathrm{H} \quad T_{a} S_{2}$ only gives a simple hexagonal hk. $\mathrm{O}$ diffraction pattern at room temperature, apart from faint steaks along $\mathrm{a}^{*}$ directions (Chevalier and Stobbs 1975). On cooling to approximately $75 \mathrm{~K}$, however, a $3 \times 3$ superlattice is observed in the patterns (Tidman et al 1974) and corresponding anomalies have been found in the susceptibility and resistivity at this temperature (Wilson et al 1974) An example of the morphology of $2 \mathrm{H} T_{a} S_{2}$ after intercalation with $\mathrm{N}_{2} \mathrm{H}_{4}$ is show in Fig 6a and the corresponding hk.O diffraction pattern is show in Fig 6b. Not only is the crystal badly distorted and filled with bubbles or voids, but the diffraction pattern suggests that there is a considerable amount of rotational disorder. This is similar to the case of intercalated $2 \mathrm{H} \mathrm{NbSe_{2 }}$ where individual bubbles (Höhlstellen) have been frequently observed (Tatlock 1977). On cooling the disordered $2 \mathrm{H} T_{a} S_{2}$ to $75 \mathrm{~K}$ the formation of the $3 \times 3$ superlattice was no longer observed. This would appear to tie in with the electrical measurements of Meyer et al (1974) on $\mathrm{NbSe}_{2}$ intercalated with ethylenediamine where the resistivity was found to vary much more smoothly in the intercalated samples than the as grown ones. 
The intercalation of $2 \mathrm{H} \mathrm{T} \mathrm{T}_{a} \mathrm{~S}_{2}$ with $\mathrm{N}_{2} \mathrm{H}_{4}$ and $\mathrm{NH}_{3}$ has also been studied by x-ray diffraction by Acrivos et al (1976) who found a phase transition near $100^{\circ} \mathrm{C}$ with this in mind high temperature experiment were attempted on room temperature intercalated crystals. It was found that on heating the crystal to $-90^{\circ} \mathrm{C}$ the disordered pattern of Fig $6 \mathrm{~b}$ transformed dramatically into the ordered $2 \times 2$ superlattice shown in Fig 7. The gross morphology of the specimens remained similar although dislocations and other defects could be observed crossing the specimen during heating. The resultant $2 \times 2$ superlattice which was stable at room temperature is reminiscent of the patterns obtained by Beal + Liang (1973) from $2 \mathrm{H} T_{a} S e_{2}$ + aniline. Yet another indication that the scattering is likely to be caused by a periodic lattice distortion rather than ordered intercalate.

\subsection{Hb Polytype}

The room temperature diffraction pattern of the mixed polytype of $T_{a} S_{2}$, $4 \mathrm{Hb}$ exhibits two characteristics $\sqrt{13} x \sqrt{13}$ superlattices (Wilson et al 1975) analogous to the low temperature pattern in the $1 \mathrm{~T}$ polytype. However two transitions are observed in the susceptibility data (Di Salvo et al 1973). One at $315 \mathrm{~K}$ corresponds to the onset of the $\sqrt{13} x \sqrt{13}$ lattice whilst the one at $\sim 20 \mathrm{k}$ corresponds to the formation of an incommensurate $a^{x} / 3$ superlattice (Tatlock 1976).

Intercalation of $4 \mathrm{Hb} T_{a} S_{2}$ at room temperature removes the $\sqrt{13} x \sqrt{13}$ lattices and gives a disordered pattern similar to the one shown for $2 \mathrm{H} T_{a} S_{2}$ in Fig $6 \mathrm{~b}$. Unlike the case of $2 \mathrm{H} T_{a} S_{2}$, however, heating to $-360 \mathrm{~K}$ does not order the pattern. This result also contrasts with the observations of Wilson et al (1975) on $1 \mathrm{~T}$ and $4 \mathrm{Hb} T_{a} S_{2}$ which had been intercalated with ethylenediamine. In both cases a diffuse $\sqrt{7} x \sqrt{7}$ pattern was obtained. It would seem likely that ethylenediamine should intercalate in a similar way to $\mathrm{N}_{2} \mathrm{H}_{4}$ and the marked differences in these results may be due to stoichiometry problems in the $4 \mathrm{Hb}$ specimens or more likely the presence of some $1 \mathrm{~T}$ crystals with the $4 \mathrm{Hb}$ specimens.

\section{Interpretation}

The most striking difference between the intercalation result in the different polytypes is the ease or otherwise with which the intercalate orders at room temperature. In all cases the crystals are badly deformed during intercalation but only the $1 \mathrm{~T}$ polytype forms an ordered superlattice at room temperature. Acrivos et al (1976) studied the reaction of $2 \mathrm{H} T_{a} S_{2}$ with hydrazine and concluded that the rate determining step for the intercalation reaction occurs on the basal surfaces with fast diffusion into the bulk near boundaries or faults. Several intermediate phases were present at room temperature but transformed into a single phase near $100^{\circ} \mathrm{C}$. This is largely confirmed by the present experiments but it would appear from the TEM observations that the intermediate phases contained pockets of hydrazine within the crystals and it is only on heating to $-100^{\circ} \mathrm{C}$ that the intercalation reaction can proceed to give an ordered superlattice. Meyer et al (1974) concluded that the relative stacking of the 
layers are changed during intercalation (e.g. 1T-> 3R stacking) and it may be that in the case of the $1 \mathrm{~T}$ polytype less energy is needed to accomplish this transformation than in the $2 \mathrm{H}$ or $6 \mathrm{Hb}$ cases. Indeed the chalcogen environment across the Van der Waals gap is predicted to be similar for the intercalated $2 \mathrm{H}$ and $4 \mathrm{H}$ cases but different in the $1 \mathrm{~T} / 3 \mathrm{R}$ case.

In an attempt to understand the changes in the periodic lattice distortion on intercalation, the various wave vectors were plotted out in the ГKM plane for the as grown $1 \mathrm{~T}$ crystals, $1 \mathrm{~T}+\mathrm{Na}, \mathrm{Li}$ etc and $1 \mathrm{~T}+\mathrm{N}_{2} \mathrm{H}_{4}$ (Fig 8) and the magnitude and directions of these vectors are listed for different temperatures in Table 1 . The most noticeable trend in the data is the way in which the magnitude of the room temperature spanning vector increases from $1 \mathrm{~T}$ through $1 \mathrm{~T}+\mathrm{N}_{2} \mathrm{H}_{4}$ to $1 \mathrm{~T}+\mathrm{Na}$. Arguments about charge transfer from the adduct to the layers could explain this effect due to a systematic change in position of the Fermi surface. This would also explain why the $\sqrt{7} x \sqrt{7}$ superlattice was occasionally observed in $1 \mathrm{~T}+\mathrm{Li}$, since incomplete intercalation may result in less charge transfer. The idea of the reverse process (i.e. charge transfer out of the layers) has also been used recently to explain the $\sqrt{13} x \sqrt{13}$ superlattice in $4 \mathrm{Hb} T_{a} S_{2}$ (Friend et al 1977). If charge were transferred from the octahedral to the trigonal prismatic layers then the Fermi surface in the octahedral layers would shrink and the $0.285 \mathrm{a}^{*}$ vector of $1 \mathrm{~T} T_{a} S_{2}$ could no longer be supported. Another possibility would then be the shorter $\mathrm{a}^{*} / \sqrt{13}$ vector. This mechanism could also offer an explanation for the production of the $\sqrt{13} x \sqrt{13}$ lattice after exposure of the intercalates to air or water vapour. If a complex were formed with the adduct then charge may also be removed from the layers causing the Fermi surface to shrink beyond that of the as grown 1T crystals thus adopting the $\sqrt{13} x \sqrt{13}$ lattice.

The charges of the superlattice vectors of $1 \mathrm{~T} T_{a} S_{2}+N_{2} H_{4}$ with temperature are quite complex, although as noted by Clarke and Williams (1977) the vectors along a* directions seem to play a central role. In the case of pure $1 \mathrm{~T} T_{a} S_{2}$ a contraction of the Fermi surface with decrease in temperature, due possibly to a temperature dependent density of states, could explain the experimental observations as suggested by Scruby et al (1975). In the $1 \mathrm{~T}$ $T_{a} S_{2}+N_{2} H_{4}$ system, however, an expansion of the Fermi surface with decrease in temperature would be required to explain the experimental results; hence other effects must also play an important role.

In the case of the trigonal prismatic $2 \mathrm{H}$ polytype a variety of spanning vectors have been suggested to explain the $3 \times 3$ superlattice observed at low temperatures. However following the charge transfer arguments of Friend et al (1977) it would seem likely that the vector shown in Fig 9 spanning the filled regions rather than hole pockets gives the most likely nesting. The formation of a 22 superlattice after intercalation is somewhat surprising if only in terms of the large change in magnitude of spanning vector from $\mathrm{a}^{*} / 3$ to $\mathrm{a}^{*} / 2$. However land structures for $1 \mathrm{~T}$ and $2 \mathrm{H} T_{a} S_{2}$ such as those of Matthew (1974) or Wexler and Wooley (1976) suggest that the lands near the Fermi level show a much greater variation in energy 
and orientation for $1 \mathrm{~T}$ rather than $2 \mathrm{H} T_{a} S_{2}$. Hence a given amount of charge transfer would have a greater effect on the portion of the Fermi surface in the $2 \mathrm{H}$ case than the $1 \mathrm{~T}$ case, in agreement with experiment.

Simple arguments of this type using only charge transfer probably understate the complexity of the problem, since the presence of intercalate must affect the land structure to some extent; nevertheless several useful trends may be predicted with this model.

\section{Conclusions}

The $1 \mathrm{~T}, 2 \mathrm{H}$ and $4 \mathrm{Hb}$ polytypes of $T_{a} S_{2}$ are intercalated with low pressure hydrazine gas at room temperature. Only the $1 \mathrm{~T}$ polytype exhibits an ordered superlattice which changes on heating or cooling. However an ordered $2 \times 2$ superlattice may be produced in $2 \mathrm{H} T_{a} S_{2}+N_{2} H_{4}$ by heating to $\sim 90^{\circ} \mathrm{C}$. The origin of all the superlattices may be explained in terms of scattering from periodic lattice distortions linked to charge density waves and comparison with other systems suggest that the observations are not due scattering directly from the intercalate. Qualitative energy arguments give useful insight into the processes involved. Here the particular band structure for the particular polytype and the changes produced on the Fermi level.

\section{Figure Captions}

Fig 1 Schematic cross section through the environmental cell used for intercalation.

Fig 2 The variation in resistivity with temperature along the a axis in $1 \mathrm{~T} T_{a} S_{2}$ and $3 \mathrm{R} T_{a} S_{2}$ (ethylenediamine) $1 / 4$

Fig 3 hk.O diffraction patterns from (a) $1 T_{2} T_{a} S_{2}+N_{2} H_{4}$ (room temp) (b) $1 T T_{a} S_{2}+N_{2} H_{4}$ (room temp)

Fig 4 (a) Analysis of the diffraction pattern of Fig 3(b) in terms of three $\frac{\sqrt{7}}{8} x \frac{\sqrt{7}}{8}$ cells (b) real space lattice corresponding to $4(\mathrm{a})$

Fig $51 T T_{a} S_{2}+N_{2} H_{4}$ (a) $80 \mathrm{~K}$ (b) on returning to room temp. (c) Streaks (d) $360 \mathrm{~K}$ (e) room temp after reaction with air/water vapour.

Fig $62 \mathrm{HT}_{a} \mathrm{~S}_{2}$ room temp. (a) crystal after intercalation (b) corresponding diffraction pattern.

Fig 7 As Fig 6(b) but after heating to 360K and then cooling to room temp.

Fig 8 ГKM phase of B7 showing all wave vectors involved in 1T superlalttices. Dashed lines indicate Scruby work.

Fig 9 Possible Fermi surfaces in $2 \mathrm{HT}_{a} \mathrm{~S}_{2}$ and $2 \mathrm{HT}_{a} \mathrm{~S}_{2}+\mathrm{N}_{2} \mathrm{H}_{4}$ showing most likely spanning vectors. 
Sussex TEM Work
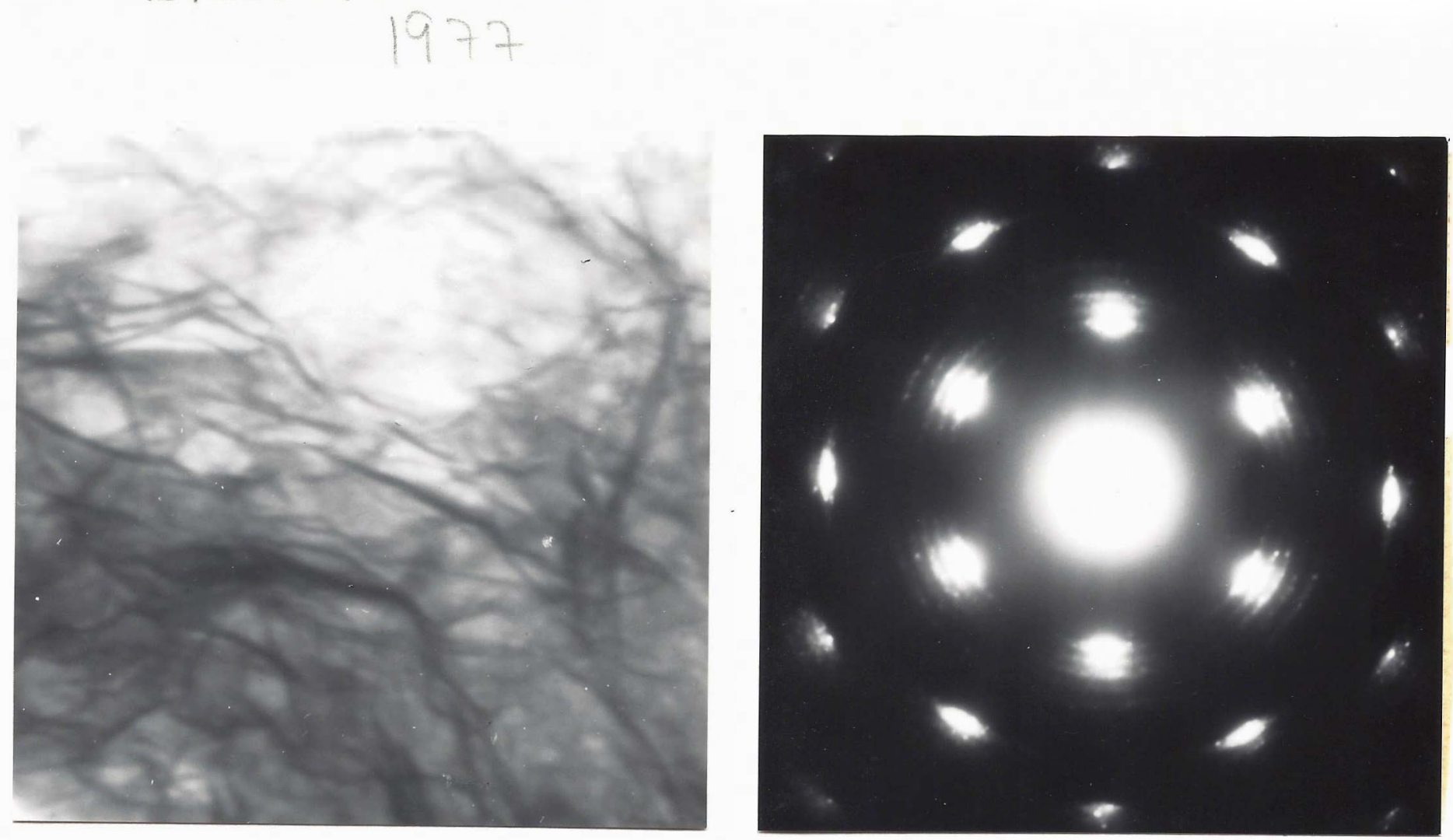

a.

Fig. $62 \mathrm{H} \mathrm{TaS}_{2}$ room temperature (a) crystal after intercalation (b) corresponding diffraction pattern.

Fig. 7 As Fig. 6(b) but after heating to $360 \mathrm{~K}$ and then cooling to room te mperature.

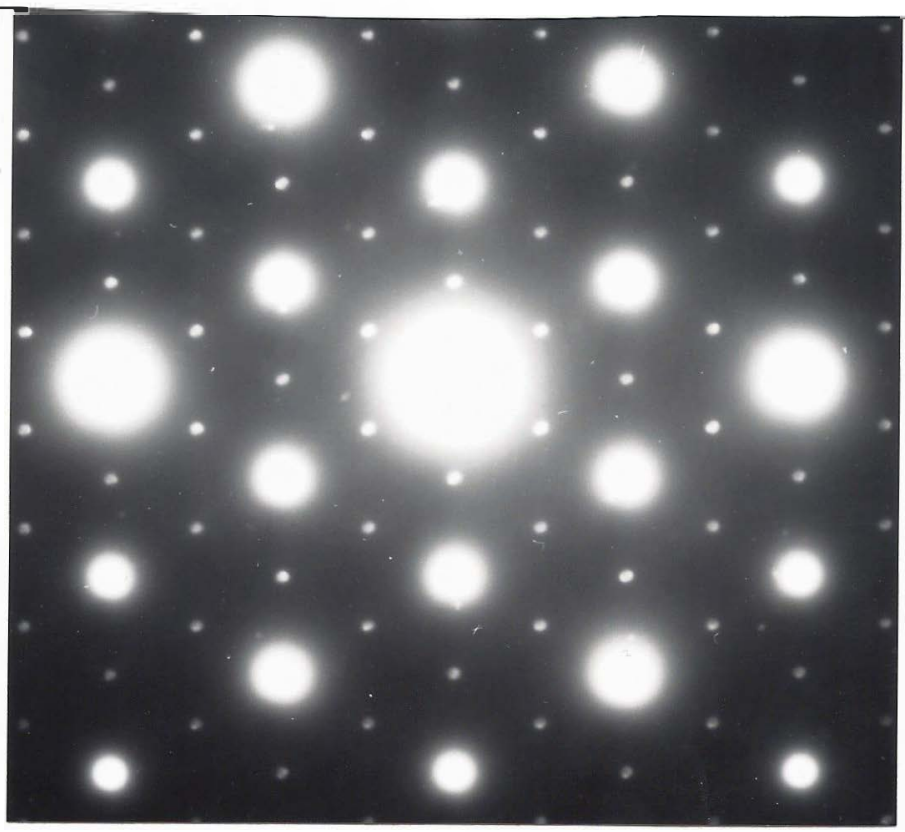



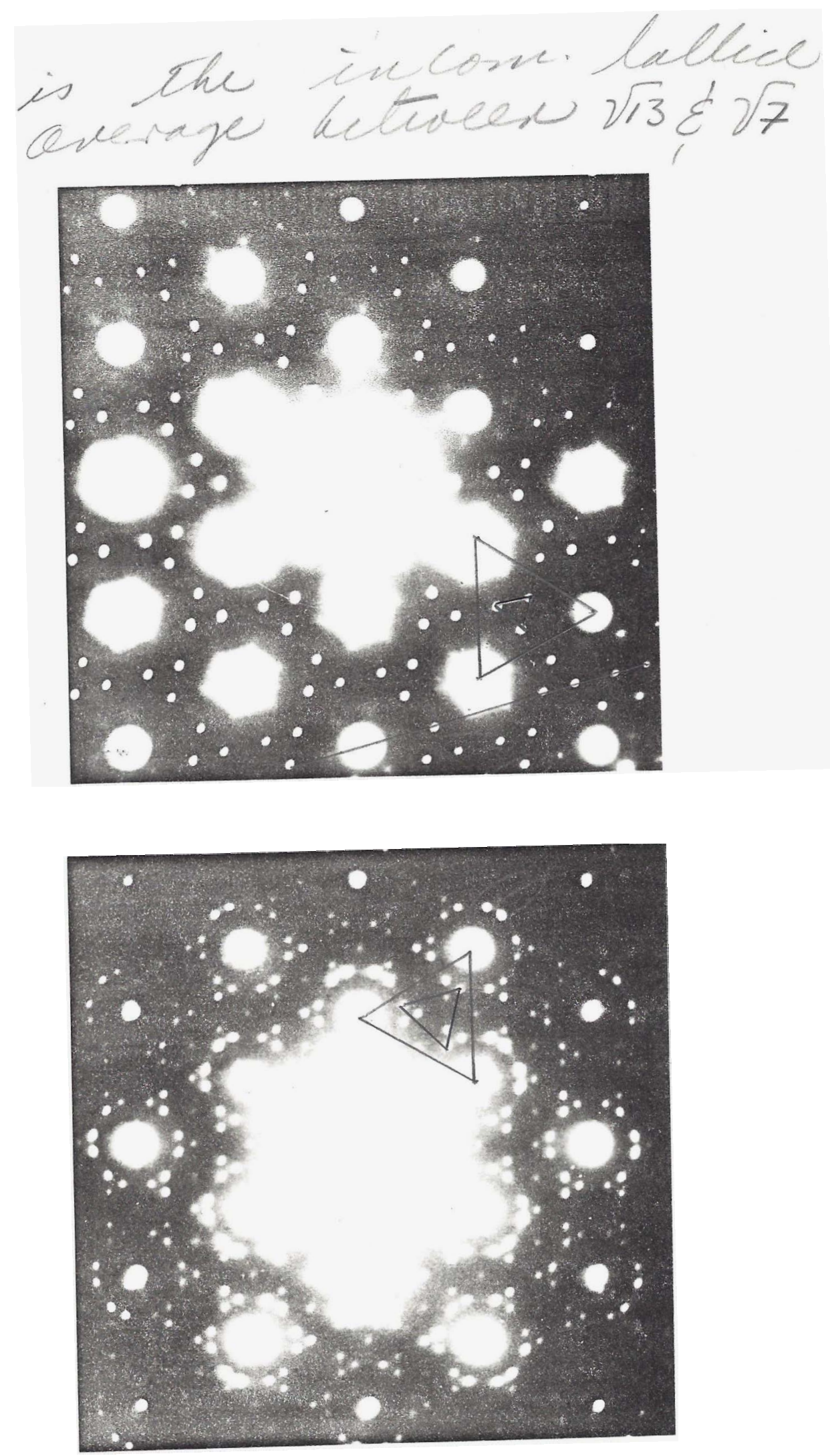

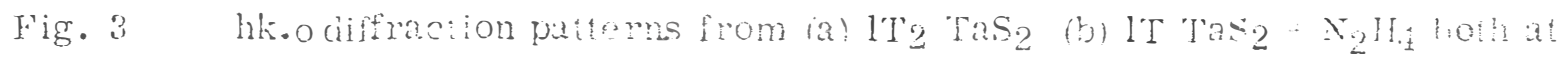
room temperiture. 


\section{References}

Acrivos, JV, Delios Co and Kurasaiki, H, 1976 Phi1 Mag 31, 1047

Beal, A R and Liang; W Y 1973, Phij. Mag 27, 1397

Carter, C B and Williams, P M, 1972, Phil Mag 26393

Chevalier, J - P AA anả Stobbs, W M 1975, Phil Mag 81733

Clark, W B and Williams, P M, 1977 Phil Mag 35883

Di Salvo, F J, Bagley, B J, Voorhoeve, J M Waszezak, J V 1973, J PhysChem Solids 2

English, C A and Venables, J A, 1971 Proc 25th Ann Meeting EMAG ed W. C. Nixon

(London : Inst of Phys) P 48.

Friend, R H, Jérome, D, Frindt, R F, Grant, A J and Yoffe, A D 1977, J Phys C 101013

Meyer, S F , Howard, R E', Stewart, G R, Acrivos, J V and Geballe, T H, 1975

J Chem. Phy's. 624411 .

Parry, G S Scruby, C B and Williams, P M 1974. Phil Mag 29601.

Scruby, C B, Williams, P M, and Parry G S, 1975, Phil Mag 31255

Tatlock, G J, 1.976, Comm on Phys 1

Tatlock, G J, 1977 J Phys D 10 L 185

Thompson, A H, Gamble, F R, and Revelli, J F, 1971. Sol Stat Comm 9981

Tidman, J P, Singh, O, Curzon, A E, and Frindt, R F, 1974. Phil Mag 3011.91

Van Landuyt, J, Van Tendeloo, G and Amelinckx, S, 1974 Phys Stat Sol 26359

Wexler, G and Wooley, A M, $1976 \mathrm{~J}$ Phys C 91185

Williams, P M, Parry, G S, and Scruby, C B, 1974 Phil Mag 29, 695

Wilson, J A and Yoffe, A D, 1969 Adv Phys 18193

Wilson, J A, Di Salvo, F J and Mahajan, S, 1974 Phys Rev Lett 32882

Wilson, J A, Di Salvo, F J and Mahajan, S 1975 Adv Phys 24117 